NASA Technical Memorandum 103685

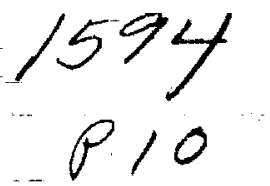

\title{
Structural Reliability Analysis of Laminated CMC Components
}

Stephen F, Duffy and Joseph L. Palko

Cleveland State University

Cleveland, Ohio

and

John P. Gyekenyesi

Lewis Research Center

Cleveland, Ohio

Prepared for the

36th International Gas Turbine and Aeroengine Congress and Exposition sponsored by the American Society of Mechanical Engineers

Orlando, Florida, June 3-6, 1991 


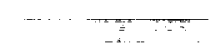 \\ a.}

$=\cdots-\ldots$

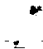




\title{
STRUCTURAL RELIABILITY ANALYSIS OF LAMINATED CYC COMPONENTS
}

\author{
Stephen F. Duffy* and Joseph L. Palko* \\ Cleveland State University \\ Department of Civil Engineering \\ Cleveland, Ohio 44115 \\ and \\ John P. Gyekenyes i \\ National Aeronautics and Space Administration \\ Lewis Research Center \\ Structural Integrity Branch \\ Cleveland, Ohio 44135
}

\section{ABSTRACT}

For laminated ceramic matrix composite (CNC) materials to realize their full potential in aerospace applications, design methods and protocols are a necessity. This paper focuses on the time-independent failure response of these materials and presents a reliability analysis associated with the initiation of matrix cracking. It highlights a public domain computer algorithm that has been coupled with the laminate analysis of a finite element code and which serves as a design aid to analyze structural components made from laminated CMC materials. Issues relevant to the effect of the size of the component are discussed, and a parameter estimation procedure is presented. The estimation procedure allows three parameters to be calculated from a failure population that has an underlying Weibull distribution.

\section{INTRODUCT ION}

Structural components produced from laminated ceramic matrix composite (CYC) materials are being considered for a broad range of aerospace applications that include propulsion subsystems in the national aerospace plane, the space shuttle main engine, and advanced gas turbine engines. Specifically, composite ceramics may be used as segmented engine liners, small missile engine turbine rotors, and exhaust nozzles. These materials will improve fuel efficiency by increasing engine temperatures and pressures, which will, in turn, generate more power and thrust. Furthermore, these materials have significant potential for raising the thrust-toweight ratio of gas turbine engines by tailoring directions of high specific reliability. The emerging composite systems, particularly those with a silicon nitride or silicon carbide matrix, can compete with metals in many demanding applications. The capabilities of Iaminated CMC prototypes have already been demonstrated at temperatures approaching $1+00{ }^{\circ} \mathrm{C}$, well beyond the operational limits of most metallic materials.

"NASA Resident Research Associate at Lewis Research Center.
Adding a second ceramic phase with an optimized interface to a brittle matrix improves fracture toughness, decreases the sensitivity of the brittle ceramic matrix to microscopic flaws, and could also improve strength. The presence of fibers in the vicinity of the crack tip modifies fracture behavior by increasing the requi red crack driving force by several mechanisms. These mechanisms include crack pinning, fiber bridging, fiber debonding, and $f$ iber pull-out. This increase in fracture toughness allows for "graceful" rather than catastrophic failure. A unidirectional ply loaded in the fiber direction retains substantial strength capacity beyond the initiation of matrix cracking despite the fact that neither of the constituents would exhibit such behavior if tested alone. First matrix cracking consistently occurs at strains greater than in the monolithic matrix material. As additional load is applied, the matrix tends to break in a series of cracks bridged by the ceranic fibers, until the ultimate strength of the composite is reached. The region of a typical stressstrain curve between the first matrix cracking and the ultimate tensile strength illustrates an intrinsic damage tolerance not present in monolithic ceramics.

Laminated CMC material systems have several mechanical characteristics which must be considered in the design of structural components. In regards to an individual ply, the most deleterious of these characteristics are low strain tolerance. Iow fracture toughness, and a large variation in failure strength in the material orientation transverse to the fiber direction. Thus analyses of components fabricated from ceramic materials require a departure from the usual deterministic philosophy of designing metallic structural components ( $i . e .$, the factor-of-safety approach). Al though the so-called size effect has been reported to be nonexistent in the fiber direction (see Dicarlo, 1989), the bulk strength of unidirectional-reinforced ply will decrease transverse to the fiber direction as the component volume increases. Since failure in the transverse direction will be dominated by the scatter in strength, statistical design approaches must be employed. These approaches must, on the one hand, allow for elevated strength, reduced variability in strength, and a diminished effect of bulk specimen size in the fiber direction, and, on the other hand, increased scatter in strength and effects of bulk size in the transverse 
direction. Simply stated, a reliability analysis must rationally account for material symmetry imposed by the reinforcement. Computational structural mechanics philosophies must emerge that address the issues of scatter in strength, size effect, and material anisotropy. There is a need for test-bed sof tware programs that incorporate stochastic design protocols, that are user friendly, that are computationally efficient, and that have flexible architectures which can readily incorporate changes in design philosophy. The C/CARES (Composi te Ceramics Analysis and Reliability Evaluation of Structures) program, which will be highlighted in this article, was developed to fulfill this need. C/CARES is a public domain computer algorithm, coupled to a general purpose finite element program, which predicts the fast fracture reliability of a laminated structural component under multiaxial loading conditions.

\section{STOCHASTIC DESICN ISSUES}

For a number of composite material systems, several authors (see for example Batdorf and Chaffarian, 1984; and $\mathrm{Wu}, 1989$ ) have reported a diminished size effect in the fiber direction; and DiCarlo (1989) has reported this effect for ceramic composites, in particular. This phenomenon is an important feature that must be addressed by any reliability model. How it is addressed depends on whether the material is modeled as a series system, a parallel system, or a combination. Current analytical practice uses finite element methods to determine the state of stress throughout the component. It is assumed that failure depends on the stress state in a component, such that deformations are not controlling design. Since failure may initiate in any of the discrete volumes (elements), it is useful to consider a component from a systems viewpoint. $A$ component comprised of discrete volumes is a series system if it fails when one of the discrete volumes fail. This approach gives rise to weakest-link theories. In a parallel system, failure of a single element does not necessarily cause the component to fail since the remaining elements may be able to sustain the load through redistribution. The parallel system approach leads to what has been referced to in the literature as "bundle" theories.

The basic principles underlying these bundle theories were originally discussed by Daniels $(19+5)$ and Coleman (1958). Their work was extended to polymer matrix composites by Rosen (1964) and Zweben (1968) Here, a relatively sof matrix serves to transfer stress between fibers and contributes little to the composite tensile strength. Hence, when a fiber breaks the load is transferred only to neighboring fibers. Their analy$s$ is is rather complex and limited to establishing bounds on the stress at which the first fiber breaks and the stress at which all the fibers are broken. Harlow and Phoenix (1978) proposed a rather abstract approach that established a closed-form solution for all the intermediate stress levels in a two-dimensional problem, and Batdorf (1982) used an approximate solution to establish the solutions for the three-dimensional problem. Batdorf's model includes the two-dimensional model as a special case. In both of the latter two models, the authors proposed that the effective Weibull modulus increases with increasing component volume. This implies a diminished size effect. However, these current bundle theories are predicated on the fact that fibers are inherently much stronger and stiffer than the matrix. In laminated CMC materials this is not always the case. The strength and stiffness of both the $f$ iber and matrix are usually closer in magnitude. For this reason bundle theories will not be considered in this paper.
We advocate the use of a weakest-link reliability theory for designing components manufactured from laminated CMC materials that do not exhibit strong size effects in specific directions. Assuming that a laminated structure behaves in a weakest-link manner allows a conservative estimate of structural reliability to be calculated. Thomas and Wetherhold (1990) point out that this assumption is equivalent to predicting the probability of the first matrix crack occurring in an individual ply. For most applications the design failure stress for a laninated material is assumed to coincide with this first ply matrix cracking because matrix cracking usually allows the fibers to oxidize at high temperatures, embrittling the composite.

Next, we address the righteousness of applying weakest-link theory to a material that in some sense does not exhibit size effects. In general, the mean strength of a sample population representing uniaxial tension test specimens can be obtained by integrating the probability of survival $P_{S}$ with respect to the applied tensile stress: that is,

$$
\tilde{\sigma}=\int_{0}^{\infty} P_{S} \mathrm{~d} \sigma
$$

Here $\vec{\sigma}$ is the mean tensile strength, $\sigma$ is the applied tensile stress, and $P_{S}$ is the probability of survival. The form of $P_{s}$ depends on the probability density function that best represents the failure data, which in turn depends on whether the structural component acts as a parallel or series system. Adopting a three-parameter. Weibull cumulative distribution function, and assuming a weakest-link system (a conservative assumption) gives the following form to Eq. (1):

$$
\vec{\sigma}=\int_{Y}^{\infty} \exp \left[-\left(\frac{\sigma-V}{\beta}\right)^{\alpha} V\right] d \sigma
$$

Here $V$ is the volume of the tensile test specimen. $\beta$ is the scale parameter, $\alpha$ is the Heibull shape parameter, and $y$ is the threshold stress. This integral has the following clased-form solution (see DeSalvo 1970):

$$
\bar{\sigma}=Y+\left[\frac{\beta}{(V)^{1 / \alpha}}\right] \Gamma\left(1+\frac{1}{\alpha}\right)
$$

which depends on volume, the Weibull parameters, and the gamma function $\Gamma$. When an argument originally outlined by Jayatilaka (1979) is followed, two uniaxial tensile specimen populations with distinctly different specimen volumes will yield different mean strengths. Associating $\bar{\sigma}_{1}$ with $v_{1}$ gives

$$
\bar{\sigma}_{1}=\gamma+\left[\frac{\beta}{\left(v_{1}\right)^{1 / \alpha}}\right] \Gamma\left(1+\frac{1}{\alpha}\right)
$$

Similarly associating $\bar{\sigma}_{2}$ with $V_{2}$ gives

$$
\bar{\sigma}_{2}=\gamma+\left[\frac{\beta}{\left(v_{2}\right)^{1 / \alpha}}\right] \Gamma\left(1+\frac{1}{\alpha}\right)
$$

If the effective mean is defined as 


$$
(\bar{\sigma})_{\text {eff }}=\bar{\sigma}-\gamma
$$

then the ratio of the effective mean strengths depends only on the specimen volume and the Weibull modulus: that is,

$$
\frac{\left(\bar{\sigma}_{1}\right)_{\text {eff }}}{\left(\bar{\sigma}_{2}\right)_{\text {eff }}}=\frac{\bar{\sigma}_{1}-\gamma}{\bar{\sigma}_{2}-\gamma}=\left(\frac{v_{2}}{v_{1}}\right)^{1 / \alpha}
$$

As the Meibull modulus of a particular material increases, the ratio of the effective mean strengths approaches unity. In this situation the material exhibits no size effect (even though the distribution of failure strength may be represented by a Heibull probability density function). From a practical standpoint, doubling the specimen size of a material whose Weibull modulus is $\approx 15$ would yield less than a 5 percent difference in the effective mean failure strengths of the two populations. We expect an elevated Weibull modulus to be associated with the strength of CYC materials in the fiber direction. Reports of an apparent lack of size effect associated with the strength in the fiber direction (see DiCarlo, 1989) could easily be an artifact of an increasing shape parameter (or small sample size). However, at this time there is an insufficient quantity of CMC failure data from which to estimate the Weibull parameters. In general, the weakest-link theory allows for diminishing size effects as the Meibull modulus increases.

\section{RELIABILITY MODEL}

The ongoing metamorphosis of ceramic material systems and the lack of standardized design data has in the past tended to minimize the emphasis on modeling. Kany structural components fabricated from ceramic materials were designed by "trial and error," since emphasis was placed on demonstrating feasibility rather than on fully understanding the processes controlling behavior. (This is understandable during periods of rapid improvements in material properties for any system.) In predicting failure behavior, there is a phi losophical division that separates analytical schools of thought into microstructural methods (usually based on principles of fracture mechanics) and phenomenological methods. Blass and Ruggles (1990) point out that analysts from the first school would design the material assuming that the constituents are distinct structural components and would consider the composite ply (or lamina) a structure in its own right. Analysts from the latter school of thought would design with the material (i.e., they would analyze structural components fabricated from the material). Rigorous fracture mechanics criteria have been proposed (e.g., Budiansky et al., 1986; and Marshall et al ., 1985) that adopt the microstructural viewpoint, but since they are all deterministic criteria, they will not be considered here. Fracture mechanics has been combined with a probabilistic Weibull analysis of failure location to determine the stress-strain behavior and subsequent work of fracture for unidirectional compos$i$ tes (e.g., Thouless and Evans, 1988; and Sutcu, 1989). However, the focus here is first matrix cracking, and we note that mature reliability-based design methods using fracture mechanics concepts will not surface until a coherent mixed-mode fracture criterion has been proposed.

The aforementioned second school of thought represents the ply (or lamina) as a homogenized material with strength properties that are determined from a number of well-planned phenomenological experiments. The authors currently embrace this philosophy, and there are practical reasons for initially adopting this viewpoint. We fully recognize that the failure characteristics of these composites are controlled by a number of local phenomena including matrix cracking, debonding and slipping between matrix and fibers, and fiber breakage, all of which strongly interact. Understanding the underlying analytical concepts associated with the microstructural viewpoint allows one to gain insight and intuition prior to constructing multiaxial failure theories that in some respect reflect the local behavior. Tensile failure in the fiber direction is dependent on these local mechanisms, and the future intent is to extend reliability methods to the constituent level in a rational and practical manner. However, a top-down approach, that is first proposing design models at the ply level, will establish viable and working design protocols. Initially adopting the bottom-up approach allows for the possibility of becoming mired in detail (experimental and analytical) when multiaxial reliabil$i t y$ analyses are conducted at the constituent level.

There is a great deal of intrinsic variability in the strength of each brittle constituent of a ceramic matrix composite, but depending on the composite system. the transverse matrix cracking strength may either be deterministic or probabilistic. Statistical models are a necessity for those composite systems which exhibit any scatter in the initiation of first matrix cracking We treat it in a probabilistic fashion, requiring that deterministic strength be a limiting case that is readily obtainable from the proposed reliability model. Predicting the reduction in reliability due to loads in the fiber direction addresses an upper bound for ply reliability in a structural design problem. Conversely, a tensile load applied transverse to the fiber direction results in failure behavior similar to a monolithic ceramic, which corresponds to the lower bound of ply reliability. Thus multiaxial design methods must be capable of predicting these two bounds as well as account for the reduction in reliability due to an in-plane shear stress, and compressive stresses in the fiber direction and transverse to the fiber direction. $A$ number of macroscopic theories exist that treat unidirectional composites as homogenized, anisotropic materials. These methods use phenomenological strength data directly without hypothesizing specific crack shapes or distributions. Theories of this genre generally are termed noninteractive if individual stress components are compared to their strengths separately. In essence, failure mechanisms are assumed not to interact, and this results in component reliability computations that are quite tractable. Work by Thomas and Wetherhold (1990), Duffy and Arnold (1990), Duffy and Manderscheid (1990), and Duffy et al. (1990) are representative of multiaxial noninteractive reliability models for anisotropic materials. In addition Wu (1989), and Hu and Goetschel (1989) have proposed simpler unidirectional reliability models for laminated composites that can be classified as noninteractive. Alternatively, one can assume that for multiaxial states of stress, failure mechanisms interact and depend on specific stochastic combinations of material strengths. Usually a failure criterion is adapted from existing polymer matrix design technologies. The probability that the criterion has been violated for a given stress state is computed using Monte Carlo methods (de Roo and Paluch, 1985) or firstorder-second-moment (FOSM) methods (Yang, 1989, and Miki et al., 1989). The interactive approach of ten results in computationally intensive reliability predictions.

In this paper a noninteractive phenomenological approach has been chosen such that a unidirectional ply is considered a two-dimensional structure, assumed to 
have five basic strengths (or failure modes). They include a tensile and compressive strength in the fiber direction, a tensile and compressive strength in the direction transverse to the fiber direction, and an in-plane shear strength. In addition each ply is discretized into individual sub-ply volumes. For reasons discussed in the previous section we assume that failure of a ply is governed by its weakest Iink (or sub-ply volume). Under this assumption, events leading to failure of a given link do not affect other links (see, for example, Batdorf and Heinisch, 1978; Wetherhold, 1983; and Cassenti, 1984); thus the reliability of the ith ply is given by the following expression

$$
R_{i}=\exp \left(-\int_{V} \Psi_{i} d V\right)
$$

where $V$ is the component volume. Here, $\psi_{i}\left(x_{j}\right)$ is the failure function per unit volume at position $x_{j}$ within the ply, given by

$\psi_{i}=\left(\frac{\left\langle\sigma_{1}-\gamma_{1}\right\rangle}{\beta_{1}}\right)^{\alpha_{1}}+\left[\frac{\left|\tau_{12}-\gamma_{2}\right|}{\beta_{2}}\right]^{\alpha_{2}}+\left[\frac{\left\langle\sigma_{2}-\gamma_{3}\right\rangle}{\beta_{3}}\right]^{\alpha_{3}}$

$$
+\left[\frac{\left\langle(-1)\left(\sigma_{1}+Y_{4}\right)\right\rangle}{\beta_{4}}\right]^{\alpha_{4}}+\left[\frac{\left\langle(-1)\left(\sigma_{2}+Y_{5}\right)\right\rangle}{\beta_{5}}\right]^{\alpha_{5}}
$$

The $\alpha$ 's associated with each term in Eq. (9) correspond to the Heibull shape parameters, the $\beta^{\prime} s$ correspond to Weibull scale parameters, and the $\gamma^{\prime} s$ correspond to the Heibull threshold stresses. In addition, $\sigma_{1}$ and $\sigma_{2}$ represent the in-plane normal stresses that are aligned with and transverse to the $f$ iber direction, respectively. Also, $\tau_{12}$ is the in-plane shear stress. The normal stresses appear twice and this allows for different failure modes to emerge in tension and compression. Note that the brackets indicate a unit step function: i.e. :

$$
\langle x\rangle=x \cdot u[x]= \begin{cases}x & x>0 \\ 0 & x \leq 0\end{cases}
$$

Inserting Eq. (9) into the volume integration given by Eq. (8) yields the reliability of the ith ply, and the probability of first ply failure for the laminate is given by the expression

$$
P_{f p f}=1-\prod_{i=1}^{n} R_{i}
$$

where $n$ is the number of plies.

This reliability model can be readily integrated with laminate analysis options available in several commercial finite element codes. A preliminary version of a public domain computer algorithm (C/CARES) that is coupled wi th MSC/NASTRAN has been developed at NASA Lewis Research Center to perform this analysis. A simple benchmark application illustrates the approach. A thin-wall tube is subjected to an internal pressure and an axial compressive load. The component is fabricated from a three-ply laminate, with a $90^{\circ} / \theta / 90^{\circ}$ layup. Here angle $\theta$ is measured relative to the longitudinal axis of the tube (see Fig. 1). An arbitrary internal pressure of $4.25 \mathrm{MPa}$ and an axial compressive stress of
87.5 MPa were applied to the tube. The Heibull parameters were also arbitrarily chosen (see Table 1). Note that the threshold stresses are taken as zero for $5 \mathrm{im}-$ plicity. In design, setting the threshold stresses equal to zero would represent a conservative assumption. The overall component reliability is depicted as a function of the midply orientation angle $(\theta)$ in Fig. 2 . The ply orientation has a decided effect on component reliability, as expected. Similar studies could demonstrate the effects of component geometry, ply thickness, load, and/or Weibul! parameters on component reliability. Hence, the C/CARES code allows the design engineer a wide latitude to optimize a component relative to a number of design parameters.

\section{PARAMETER ESTIMATION}

We anticipate that laminated CYC materials will exhibit threshold behavior, at least in the fiber direction. Hence, a three-parameter Weibull distribution is used in the stochastic fallure analysis of the components. The threshold stress parameter is included to allow for zero probability of failure when the load is below a predetermined level. The three-parameter distribution has been somewhat ignored due to difficulties encountered in extracting the parameters from experimenta! data. Several authors (including Weibull, 1939; Weil and Daniel. 1964; and Schneider and Palazot to. 1979) have proposed estimation methods for the threeparameter distribution. For various reasons, these techniques have not been widely accepted. However, Cooper (1988) recently proposed a nonlinear regression method to estimate parameters. Regression analysis postulates a relationship between two variables. In an experiment, typically one variable can be controlled (the independent variable) while the response variable (or dependent variable) is uncontrolled. In simple failure experiments the material dictates the strength at failure, indicating that the failure stress is the response variable. The ranked probability of failure $y_{i}$ can be controlled by the experimentalist since it is functionally dependent on the sample size $\mathrm{N}$. If the observed failure stresses $\left(\sigma_{1}, \sigma_{2}, \sigma_{3}, \ldots . ., \sigma_{1}\right)$ are placed in ascending order, then

$$
\mathscr{P}_{i}\left(\sigma_{i}\right)=\frac{i-0.3}{N+0.4}
$$

Clearly one can influence the ranked probability for a given stress level by increasing or decreasing the sarple size. Cooper's procedure adopts this philosophy, and the specimen failure stress is treated as the dependent variable. The associated ranked probability of failure is assumed to be the independent variable. The basic three-parameter Weibull expression for probability of failure can be expressed as

$$
\tilde{\sigma}_{i}=\tilde{Y}+\tilde{\beta}\left[\ln \left(\frac{1}{1-\tilde{P}_{i}}\right)\right]^{1 / \tilde{\alpha}}
$$

where $\tilde{\sigma}_{i}$ is an estimate of the dependent variable, and $\tilde{\gamma}, \tilde{\beta}$, and $\tilde{\alpha}$ are estimates of the threshold parameter, the characteristic strength, and the shape parameter, respectively. Defining

$$
\delta_{i}=\vec{\sigma}_{i}-\sigma_{i}
$$

as the $i$ th residual, where as before $\sigma_{i}$ is the ith failure stress, then 


$$
\sum_{i=1}^{N}\left(\delta_{i}\right)^{2}=\sum_{i=1}^{N}\left(\tilde{y}+\tilde{\beta} w_{i}^{1 / \bar{\alpha}}-\sigma_{i}\right)^{2}
$$

where we adopt Cooper's notation and take

$$
w_{i}=\ln \left(\frac{1}{1-\mathscr{P}_{i}}\right)
$$

Setting the partial derivatives of the sum of the squares of the residuals with respect to $\bar{\gamma}, \tilde{\beta}$, and $\tilde{a}$ equal to zero yields the following three expressions:

$\tilde{\beta}=\frac{N\left[\sum_{i=2}^{N} \sigma_{i}\left(w_{i}\right)^{1 / \tilde{\alpha}}\right]-\left[\sum_{i=1}^{N} \sigma_{i}\right]\left[\sum_{i=1}^{N}\left(w_{i}\right)^{1 / \bar{\alpha}}\right]}{N \sum_{i=1}^{N}\left(w_{i}\right)^{2 / \tilde{\alpha}}-\left[\sum_{i=1}^{N}\left(w_{i}\right)^{1 / \tilde{\alpha}}\right]\left[\sum_{i=1}^{N}\left(w_{i}\right)^{1 / \tilde{\alpha}}\right]}$

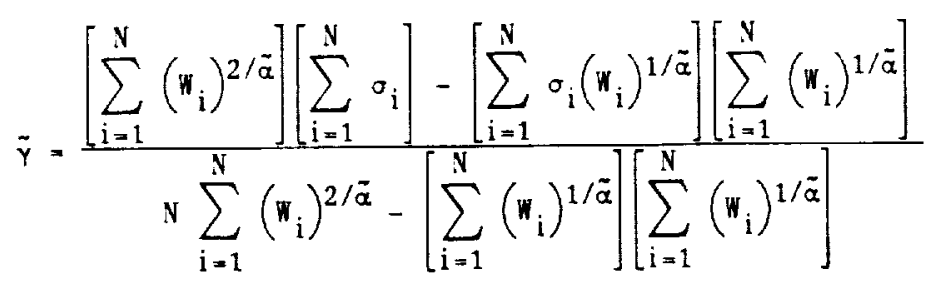

and

$$
\begin{aligned}
\sum_{i=1}^{N} \sigma_{i}\left(w_{i}\right)^{1 / \bar{\alpha}_{1 n}\left(w_{i}\right)=} & \tilde{y} \sum_{i=1}^{N}\left(w_{i}\right)^{1 / \bar{\alpha}_{\ln }\left(w_{i}\right)} \\
& +\tilde{\beta} \sum_{i=1}^{N}\left(w_{i}\right)^{2 / \tilde{\alpha} \ln \left(w_{i}\right)}
\end{aligned}
$$

in terms of the parameter estimates. The solution of this system of equations is iterative. One assumes an initial value for $\tilde{\alpha}$ (a small value, usually equal

to 1), computes $\tilde{\beta}$ from Eq. (17) and $\tilde{Y}$ from Eq. (18). These values of the parameter estimates are then inserted into Eq. (19), and this expression is checked to see if it satisfies some predetermined tolerance. If Eq. (19) is not satisfied, $\alpha$ is increased and a new iteration is conducted. This procedure continues until a set of parameter estimates are determined that satisfy Eqs. (17) to (19).

Currently we do not have enough CMC failure data to estimate Weibull parameters for a given material orientation. So to illustrate the technique, parameter estimates were determined for two-parameter and threeparameter distributions from a failure population representing a monolithic ceramic (alumina) reported by Quinn (1989). The failure data and specimen geometry are shown in Table 2 and Fig. 3 . Figure 4 is a plot of probability of failure versus failure stress for the data. The straight line represents the two-parameter fit to the data, using Quinn's (1989) values for the shape and scale parameters. The nonlinear curve represents the three-parameter fit to the data. Note that the three-parameter distribution is more efficient in predicting the failure data in the high reliability region.

\section{SUMMARY AND FUTURE DIRECTIONS}

In this paper we discuss stochastic issues related to size effects in the fiber direction of a unidirectional CMC material. In addition, we present a reliability model along with a simple application that highlighted the C/CARES computer algarithm. (This public domain algorithm is capable of predicting component reliability from the state of stress and temperature distribution within the component.) The authors anticipate that CMC materials will exhibit threshold behavior, hence a nonlinear regression analys is was outlined to determine three parameters for a Heibull distribution from failure data.

Ceramic material systems will play a significant role in future elevated-temperature applications. To this end, there are a number of issues that must be addressed by the structural mechanics research community. We begin by pointing out that total failure of an individual ply effectively reduces the overall laminate stiffness. This causes local redistribution of the load to adjacent layers. In addition, delamination between plies relaxes the constraining effects among layers. allowing in-plane strains to vary in a stepwise fashion within a laminate. These effects require the development of rational load redistribution schemes. It is also apparent that before ceramics are used as structural components in harsh service environments, thoughtful consideration should be given to reliability degradation due to time-dependent phenomena. Thus, issues germane to component life, such as cyclic fatigue and creep behavior, must be addressed analytically. Computational strategies are needed to extend current methods of analysis from subcritical crack growth and creep rupture to laminated C.YC materials that are subject to multiaxial states of stress.

An important aspect that has not been addressed in detail is the effect of a rising R-curve behavior, where fracture toughness is a function of crack size. Clearly fiber-toughened matrices have process zones around the crack tip. Within this zone, energy dissipates locally, which develops a damage tolerance by increasing the resistance to crack growth with crack extension. Failure of materials exhibiting R-curve behavior would depend on the rate at which resistance increases with crack growth. During crack extension this behavior would modify the strength distribution. Modeling ef forts by Kendall et al. (1986) and others have accounted for this behavior in monolithic ceramics, and it is reasonable to extend their work to ceramic composite material systems. Furthermore, if ceramic materials mimic ductile failure locally, cyclic fatigue may become a design issue. Under cyclic loads, the process zone advances as the crack tip extends; therefore, brittle fracture mechanics way need to be modified to account for pseudoductile fracture. Hence, application of modified metallic fatigue analyses may be a distinct possibility.

In addition, recent progress in processing ceramic material systems has not been matched by mechanical testing efforts. There is a definite need for experiments that support the development of reliability models. Initially this effort should include experiments that test the fundamental concepts (e.g. , quant ifying the size effect in the fiber direction) within the framework of current stochastic models. For example, probing experiments could be conducted along various biaxial load paths to establish level surfaces of reliability in a particular two-dimensional stress space (similar to probing yield surfaces in metals). One 
could then verify such concepts as the maximum stress response which is of ten assumed in the noninteractive reliability models proposed for these materials. After establishing a theoretical framework, characterization tests should then be conducted to provide the functional dependence of model parameters with respect to temperature and environment. Finally data from structural tests that are multiaxial (and possibly nonisothermal) would be used to challenge the predictive capabilities of models through comparison to benchmark response data. These tests involve nonhomogeneous fields of stress, deformation, and temperature, and would include two-bar tests as well as plate and shell structures. Results from structural testing provide feedback for subsequent modification, but ad hoc models result in the absence of structured interaction between the experimentalist and the theoretician. The validity of these models are then forever open to question. Furthermore, we cannot overemphasize that this kind of testing supports methods for designing components, not the materials. Currently this effort is hampered by the quality and scarcity of data (note the lack of failure data necessary to estimate composi te Weibull parameters). Finally, ceramic properties pertinent to structural design (which include stochastic paraneters) vary with test methods. The mechanics research community is beginning to realize this, and a consensus is beginning to form regarding standards. However, we wish to underscore the fundamental need for experimental programs that are relevant to structural mechanics issues.

In closing, we recognize that when failure is less sensitive to imperfections in the material, stochastic methods may not be as essential. Yet, trends in design protocols are moving in the direction of probabilistic analyses (even for metals) and away from the simplistic safety-factor approach. In this sense, brittle ceranics will serve as prototypical materials in the study and development of reliability models that will act as the basis of future design codes.

\section{REFERENCES}

Batdorf, S.B., and Heinisch, H.L., Jr., 1978, "Weakest Link Theory Reformulation for Arbitrary Fracture Criterion," Journal of the American Ceramic Society, Vol. 61, pp. 355-358.

Batdorf, S.B., 1982, "Tensile Strength of Unidirec. tionally Reinforced Composites - I," Journal of Reinforced Plastics and Composites, VoI.1, No. 2, pp. 153-164.

Batdorf, S.B., and Chaffarian, R., 1984, "Size Effect and Strength Variability of Unidirectional Composites," International Iournal of Fracture, Vol. 26, pp. $113-123$.

Blass, J.J., and Ruggles, M.B., 1990, "Design Methodology Needs for Fiber-Reinforced Ceramic Heat Exchangers," ORNL/TM-11012, Oak Ridge National Lab.

Budiansky, B., Hutchinson, J.W., and Evans, A.C., 1986, "Matrix Fracture in Fiber-Reinforced Ceramics," Iournal of the Mechanics and Physics of Solids, Vol. 34 , No. 2, pp. 167-189.

Cassenti, B.N., 1984, "Probabilistic Static Failure of Composite Material," AIAA Journal, Vol. 22, No. 1 , pp. $103-110$.

Coleman, B.D., 1958, "On the Strength of Classical Fibers and Fiber Bundles," Lournal of the Mechanics and Physics of Solids, Vol. 7, No, 1, pp. 66-70.
Cooper, N.R., 1988, "Probabilistic Failure Prediction of Rocket Motor Components," PhD Thesis, Royal Military College of Science. (Avail. Univ. Microfilms Inc.)

Daniels, H.E., 1945, "The Statistical Theory of the Strength of Bundles of Threads," Proceedings of the Royal Society of London, Series A, Vol. 183, No. 995, pp. 405-435.

de Roo, P., and Paluch, B., 1985, "Application of a Multiaxial Probabilistic Failure Criterion to a Unidirectional Composite," Developments in the Science and Technology of Composite Materials. A.R. Bunsell, P. Lamicq, and A. Massiah, eds., Assoc i ation Européenne des Materiaux Composites, Bordeaux, pp. $328-334$

DeSalvo, G.J., 1970. "Theory and Structural Design Applications of Weibull Statistics," WANL-TME-2688, Westinghouse dstronuclear Laboratory.

DiCarlo. J.A., 1989, "CYC's for the Long Run," Idvanced Materials and Processes. Vol. 135, Issue 6. pp. $+1-4 t$

Duffy, S.F., and Arnold, S.M., 1990, "Noninieractive Macroscopic Reliability Wodel for Whisker Reinforced Ceramic Composites," Lournal of Composite Vaterials. Vol. 24 , vo. 3, pp. 293-308.

Duffy, S.F., and Yandersheid, J..., 1990, "Voninteractive Macroscopic Reliability Vodel for Ceranic Matrix Composites with Orthotropic Material Symmetry," Journal of Engineering for Gas Turbines and Power. Vol. 112, No. 4, pp. 507-511,

Duffy, S.F., Hetherhoid, R.C., and Jain, L.K., igS0 "Extension of a Noninteractive Reliability Model for Ceramic Matrix Composites." NASA CR-18526-

Harlow, D.C., and Phoenix, S.L., 1978, "The Chain-oi Bundles Probability Mode! for the Strength of Fibrous Materials - 1. Analysis and Conjectures," Lournal of Composite Materials, Vol. 12, No, 2, pp. 195-214.

Hu, T.C., and Goetschel, D.B., 1989, "The Application of the Heibull Strength Theory to Advanced Composite Materials," Tomorrow's Materials: Tuday, Vol. 1, Proceedings of the 34 th International SAMPE Symposium and Exhibition, G.A. Zakrzewski, et al,, eds., SAMPE. Covina, CA, pp. 585-599.

Jayat llaka, A.S., 1979, Fracture of Engineering Brittle Materials, Applied Science Publishers, London, England, pp. 249-257.

Kendall, K., Alford, X.Y., Tan, S.R., and Bircha!l, J.D., 1986, "Influence of Toughness on Weibull Modulus of Ceramic Bending Strength," Journal of Materials Research, Vol. 1, No. 1, pp. 120-123.

Marshall, D.B., Cox, B.N., and Evans, A.G., 1985, "The Mechanics of Matrix Cracking in Brittle Matrix Fiber Composites," Acta Metallurgica, Vol. 33, No. 11, 1985; pp. 2013-2021.

Miki, M., Murotsu, Y., Tanaka, T., and Shao, S., "Reliability of the Strength of Unidirectional Fibrous Composites," 30th Structures. Structural Dynamics and Materials Conference, Part 2, AIA, Washington, D.C., pp. 1032-1040. 
Quinn, G.D., 1989, "Flexure Strength of Advanced Ceramics - A Round Robin Exercise," MTL TR-89-62 (Avail. NTIS, AD-A212101).

Rosen, B.W., 1964, "Tensile Failure of Fibrous Composites," AlAA Journal, Vol. 2, No. 11, pp. 1985-1991.

Schneider, D., and Palazotto, A.N., 1979, "A Technique for Evaluating a Unique Set of Three Neibull Parameters Considering Composite Materials, "Fibre Science and Technology, Vol. 12, No. 4, pp. 269-281.

Sutcu, M., 1989, "Weibull Statistics Applied to Fiber Failure in Ceramic Composites and Work of Fracture." dcta Metallurgica, Vol. 37, No. 2, pp. 651-661.

Thomas, D.J., and Wetherhold, R.C., 1990, "Reliability Analys is of Continuous Fiber Composite Laminates," NASA CR-185265.

Thouless, M.D., and Evans, A.G., 1988, "Effects of PullOut on the Mechanical Properties of Ceramic-Matrix Composites," Acta Metallurgica, Vol. 36, No. 3, pp. $517-522$.
Weibull, W.A., 1939, "Statistical Theory of the Strength of Materials," Ingeniors Vetenskaps Akadamien Handl ingar, No. 151 .

Weil, N.A., and Daniel, I.M., 1964, ".nalysis of Fracture Probabilities in Nonuniformly Stressed Brittle Materials," Journal of the American Ceramic Society. Vol. 47, No. 6, pp. 268-274.

Wetherhold, R.C., 1983, "Statistics of Fracture of Composite Material Under Multiaxial Loading," PhD Dissertation, University of Delaware.

Wu, H.F., 1989, "Statistical Analysis of Tensile Strength of ARALL Laminates." Lournal of Composite Materials, Vol. 23, No. 10, pp. 1065-1080.

Yang, L., 1989, "Reliability of Composite Laminates." Mechanics of Structures and Machines, Vol. 16, No. 4 , pp. 523-536.

Zweben, C., 1968, "Tensile Failure of Fiber Composites," AIA Journal, Vol., 6, No. 12, pp. 2325-2331.

TABLE 1. - COMPOSITE WEIBULL PARAMETERS FOR THIN-WALL TUBE

[Weibull threshold stress, $\left.Y_{i} \equiv 0.\right]$

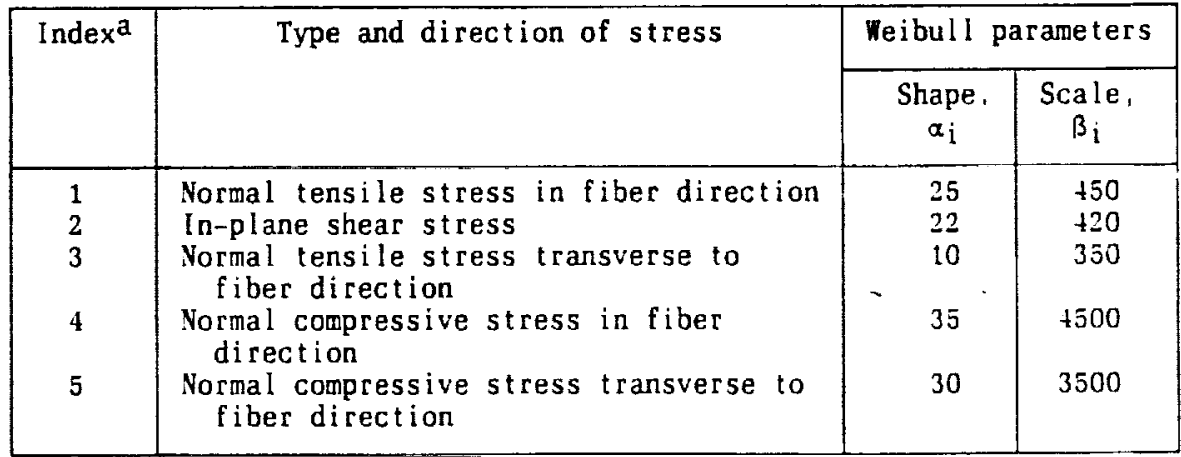

andices correspond to subscripts in Eq. (9).

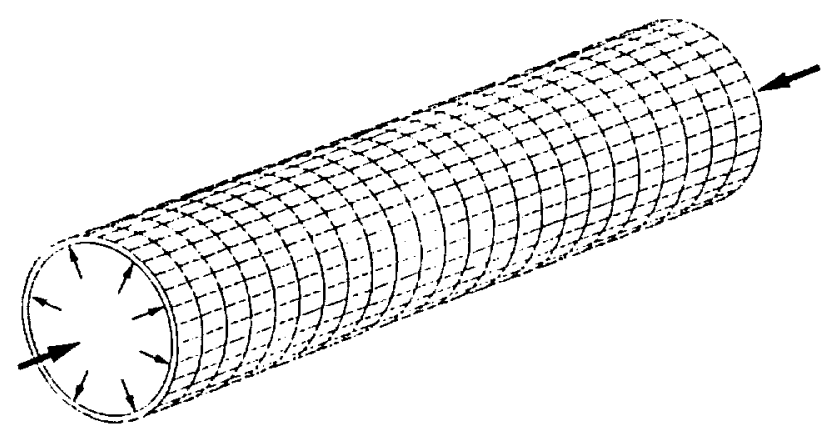

Fig. 1. Finite element mesh of thin-wall tube with three-ply $\left(90^{\circ} / 6 / 90^{\circ}\right)$ layup. Internal pressure, 4.25 MPa; axial compressive stress, $87.5 \mathrm{MPa}$. 


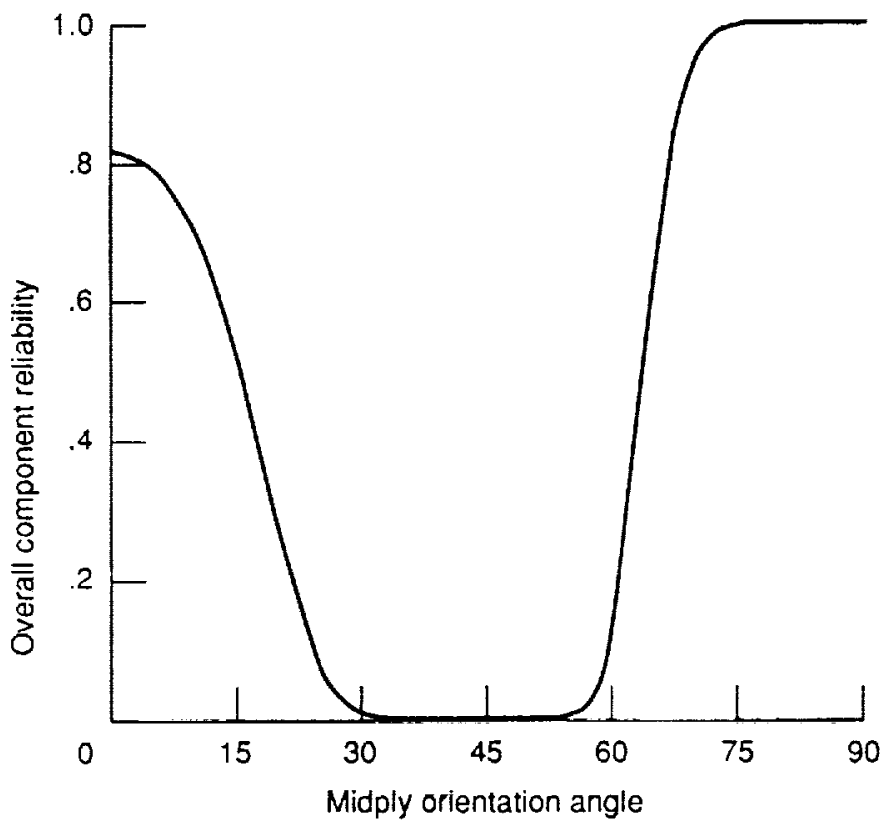

Fig. 2. Component reliability versus midply orientation angle for thin wall tube.

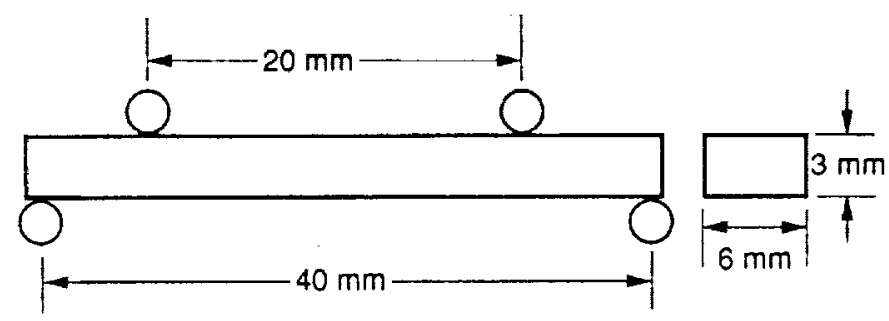

Fig. 3. Monolithic alumina specimen geometry.

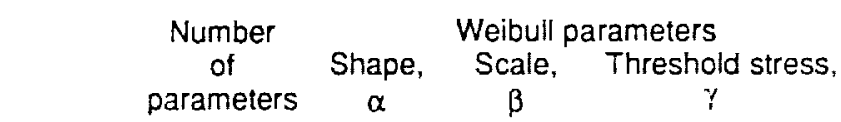

\begin{tabular}{lllll} 
& 3 & 1.15 & 803.41 & 298.48 \\
\hline & 2 & 13.2 & 376.0 & \\
\hline & Data (35 points) & &
\end{tabular}

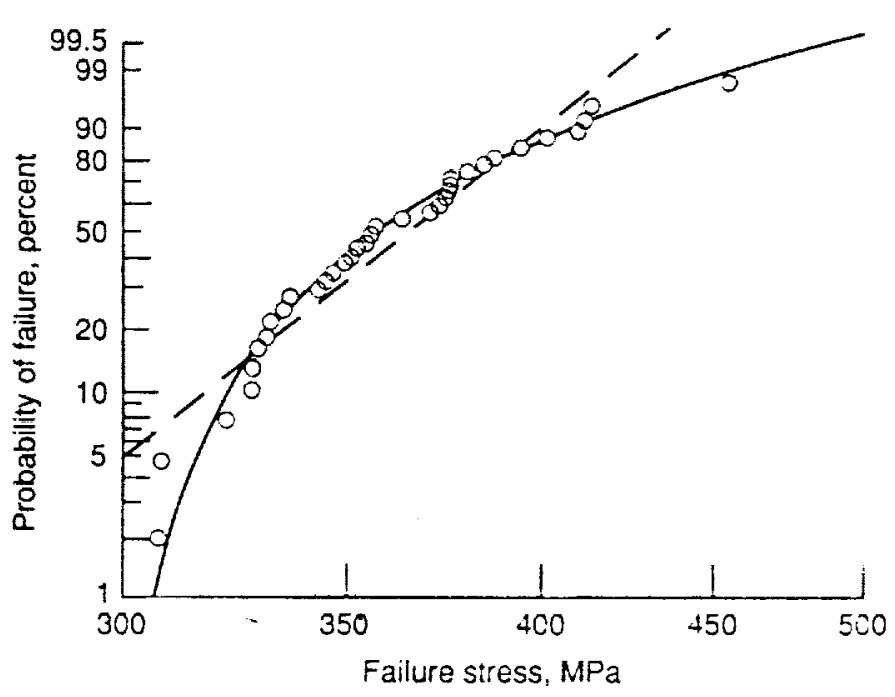

Fig. 4. Two-parameter and three-parameter distributions determined from the alumina failure.

TABLE 2. - MONOLITHIC ALLMINA FAILURE DATA

\begin{tabular}{|c|c|c|c|c|c|c|c|}
\hline $\begin{array}{l}\text { Speci- } \\
\text { men } \\
\text { number }\end{array}$ & $\begin{array}{c}\text { Stress } \\
\mathrm{MPa}\end{array}$ & $\begin{array}{l}\text { Speci - } \\
\text { men } \\
\text { number }\end{array}$ & $\begin{array}{c}\text { Stress, } \\
\mathrm{MPa}\end{array}$ & $\begin{array}{l}\text { Speci- } \\
\text { men } \\
\text { number }\end{array}$ & $\begin{array}{c}\text { Stress, } \\
\mathrm{MPa}\end{array}$ & $\begin{array}{l}\text { Speci- } \\
\text { men } \\
\text { number }\end{array}$ & $\begin{array}{c}\text { Stress } \\
\mathrm{MPa}\end{array}$ \\
\hline $\begin{array}{l}1 \\
2 \\
3 \\
4 \\
5 \\
6 \\
7 \\
8 \\
9\end{array}$ & $\begin{array}{l}307 \\
308 \\
322 \\
328 \\
328 \\
329 \\
331 \\
332 \\
335\end{array}$ & $\begin{array}{l}10 \\
11 \\
12 \\
13 \\
14 \\
15 \\
16 \\
17 \\
18\end{array}$ & $\begin{array}{l}337 \\
343 \\
345 \\
347 \\
350 \\
352 \\
353 \\
355 \\
356\end{array}$ & $\begin{array}{l}19 \\
20 \\
21 \\
22 \\
23 \\
24 \\
25 \\
26 \\
27\end{array}$ & $\begin{array}{l}357 \\
364 \\
371 \\
373 \\
374 \\
375 \\
376 \\
376 \\
381\end{array}$ & $\begin{array}{l}28 \\
29 \\
30 \\
31 \\
32 \\
33 \\
34 \\
35\end{array}$ & $\begin{array}{l}385 \\
388 \\
395 \\
402 \\
+11 \\
+13 \\
+15 \\
+56\end{array}$ \\
\hline
\end{tabular}

a For specimen shown in Fig. 3 . 


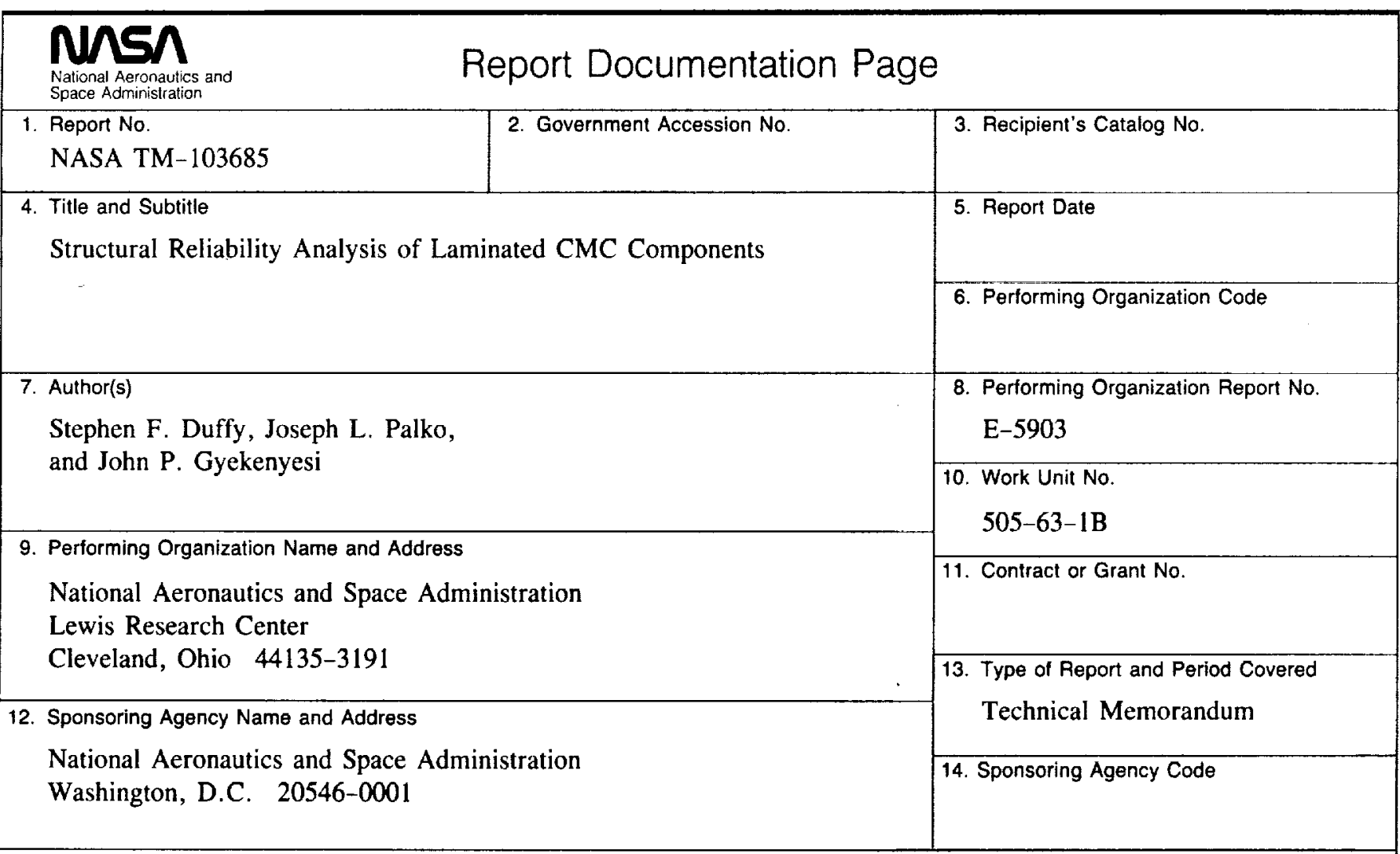

15. Supplementary Notes

Prepared for the 36th International Gas Turbine and Aeroengine Congress and Exposition sponsored by the American Society of Mechanical Engineers, Orlando, Florida, June 3-6, 1991. Stephen F. Duffy and Joseph L. Palko, Department of Civil Engineering, Cleveland State University, Cleveland, Ohio 44115 and NASA Resident Research Associates at Lewis Research Center. John P. Gyekenyesi, NASA Lewis Research Center, Responsible person, Stephèn F. Duffy, (216) 433-5626.

16. Abstract

For laminated ceramic matrix composite (CMC) materials to realize their full potential in aerospace applications, design methods and protocols are a necessity. This paper focuses on the time-independent failure response of these materials and presents a reliability analysis associated with the initiation of matrix cracking. It highlights a public domain computer algorithm that has been coupled with the laminate analysis of a finite element code and which serves as a design aid to analyze structural components made from laminated CMC materials. Issues relevant to the effect of the size of the component are discussed, and a parameter estimation procedure is presented. The estimation procedure allows three parameters to be calculated from a failure population that has an underlying Weibull distribution.

\begin{tabular}{|l|l|l|l|}
\hline $\begin{array}{l}\text { 17. Key Words (Suggested by Author(s)) } \\
\text { Reliability; Composites; Ceramics; Parameter estimates; } \\
\text { Nonlinear regression; Size effects }\end{array}$ & $\begin{array}{r}\text { 18. Distribution Statement } \\
\text { Unclassified - Unlimited } \\
\text { Subject Category } 27\end{array}$ \\
\hline $\begin{array}{l}\text { 19. Security Classif. (of this report) } \\
\text { Unclassified }\end{array}$ & $\begin{array}{c}\text { 20. Security Classif. (of this page) } \\
\text { Unclassified }\end{array}$ & $\begin{array}{c}21 . \text { No. of pages } \\
10\end{array}$ & $\begin{array}{c}22 . \text { Price }^{\circ} \\
\text { A02 }\end{array}$ \\
\hline
\end{tabular}

NASA FORM 1626 OCT $86 \quad$ *For sale by the National Technical Information Service, Springfield, Virginia 22161 
